\title{
Two-Dimensional Tableaux
}

\author{
David R. Gilbert
}

\begin{abstract}
We present two-dimensional tableau systems for the actuality, fixedly, and $\uparrow$ operators. All systems are proved sound and complete with respect to a two-dimensional semantics. In addition, some issues regarding decidability are discussed.
\end{abstract}

\section{Introduction}

This article presents prefixed tableau systems ${ }^{1}$ for some modal logics that contain special operators meant to formalize the notions of "actually" and "fixedly". Specifically, we will consider propositional modal logics that are enriched by way of the addition of the sentential operators @ and F, as well as for the Vlach-operator $\uparrow .{ }^{2}$ Our tableau systems will be doubly-prefixed. This corresponds to the use of double indexing in the two-dimensional semantics we will give for these operators, and with respect to which our proof systems will be proved sound and complete.

The actuality operator is a frequently employed device enabling some of the expressive inadequacies of quantified modal logic to be shored-up. ${ }^{3}$ The fixedly operator was originally introduced by Davies and Humberstone [10] as a means of exploring different kinds of necessities. This idea has been expanded upon in recent years, and the combination of the actuality and fixedly operators has been employed in efforts to formally analyze notions relating to a priority, specifically the contingent a priori as well as the

\footnotetext{
${ }^{1}$ Our notation is most similar to [18] and [15], which has its origins in [12].

${ }^{2}$ In [7], Ref is used as the symbol for $\uparrow$. In [26], it is $\times$. We choose to use the $\uparrow$ notation as we think it more closely coincides with the contemporary formal literature on this subject. For details on Vlach-operators, one can consult [16] or [6]. They originate in [30].

${ }^{3}$ See, for example, [22] and [4] for more on the expressive capabilities of first-order modal logics extended with the actuality and other, similar, operators. As we discuss below, in the propositional case the actuality operator is redundant.
}

Australasian Journal of Logic (13:7) 2016, Article no. 1 
necessary a posteriori. ${ }^{4}$ To our knowledge, the formal aspects of the logic of fixedly remains laregly unexplored. Davies provided a detailed examination of the behaviour of fixedly within the context of S5, where it turns out to be eliminable [8]. It is our hope that this paper begins to address some of the remaining issues surrounding the fixedly operator. In fact, providing a completeness proof for the logic of fixedly is a primary motivation of this paper.

Lastly, we also include $\uparrow$ in some of the languages in this paper. As pointed out in Cresswell [7], this operator increases the expressivity of our modal language by allowing us to store a new reference index while analyzing a formula. In addition, adding this operator is practically uncomplicated, as it will turn out that once the formal apparatus for @ and F is in place, it is straightforward to introduce $\uparrow$ as well.

The paper will begin by considering @ in isolation. In Section 2 we introduce the language of actuality logic, and describe a two-dimensional semantics appropriate for the study of actuality. Section 3 presents the double-indexed prefixed tableau rules for @, as well as the pertinent notions of proof. The tableau rules are proved to be sound and complete with respect to the two-dimensional semantics of section 2. In Section 4 a decision procedure for the actuality tableaux is discussed, and a decidability proof provided. Section 5 then presents tableau rules for the fixedly operator. Soundness and completeness proofs are provided while paying attention to the added complications introduced by F. Lastly, in Section 6 , rules for $\uparrow$ are provided, and soundness and completeness proofs are given for the logic with just @ and $\uparrow$, as well as for the full logic, with all three operators. Further issues concerning decidability are briefly discussed in the appendix.

\section{$1.1 \quad$ Related Work}

While the actuality operator has been studied for some time, in the case of propositional modal logic, adding only the actuality operator does not increase the expressive capabilities of the formal language, a result given in [21] that mirrors the case for the "now" operator in temporal logics, originally proved by Hans Kamp, in [23]. Thus, in a propositional setting, the primary interest of the actuality operator lies in the way in which it combines with other new operators, in this case other two-dimensional operators.

In addition, as mentioned above, an actuality operator is utilized in

\footnotetext{
${ }^{4}$ There is a large, and growing literature on these issues. For greater detail on the philosophical underpinnings of these logics, one might consult, for example, [5], [11], [28], and [27]. For an introduction to these issues, the last chapter of [26] is a good source.
} 
studies of epistemic two-dimensional semantics. There has been some recent work aimed at formalizing these ideas. In particular, Restall [24] gives a cutfree hypersequent calculus that captures the logics discussed by Davies and Humberstone in [10]. An interesting, and more semantic approach to these issues is described in [17]. The operators discussed in this paper, though not motivated by the same philosophical concerns, are closely related.

Lastly, the operators we consider all have analogues in hybrid logic. However, it is usually the case that migrating to a hybrid framework greatly increases the strength of the logics being considered. For example, the cost of adding binders (akin to the Vlach operator $\uparrow$ discussed here) to basic hybrid languages is usually undecidability, even in basic cases where, for instance, only one state variable is used. As is shown in [29], the real source of the undecidability in hybrid logics with binders is the interaction between the binder and the modal operators, not the @ operator. One can also show how the question of the decidability of these logics is related to the number of modal operators occurring between the binding of its variable and its use. This was recently investigated in the framework of coalgebraic modal logics as well as Kripke semantics in [19]. As is shown in the appendix, the logics we consider do not suffer from these drawbacks. Thus, this paper can also be seen as an effort to approach particular logics, which are quite expressive, from below, without adopting the full power of hybrid logics, even relatively weak ones.

\section{Two-Dimensional Semantics and the Language of Actuality}

\section{$2.1 \quad$ Language}

Where $\Phi$ is a countable set of propositional variables, let $\mathcal{L}(\Phi)+@$ (often just $\mathcal{L}+@$ when unambiguous) denote the language of propositional modal actuality logic that will be employed throughout this paper. The language has the standard connectives and modal operators, as well as the actuality operator@.

$$
\varphi::=p|\neg \varphi| \varphi \wedge \psi|\varphi \vee \psi| \varphi \rightarrow \psi|\square \varphi| @ \varphi
$$

In the second part of the paper, we consider logics containing a fixedly operator F. For these new operators, one simply supplements the normal formation rules with the following: if $\varphi$ is a wff, then so is @ $\varphi$; if $\varphi$ is a wff, 
then so is $F \varphi$. Finally, when we add $\uparrow$, we have the condition that if $\varphi$ is a wff, then so is $\uparrow \varphi$.

\subsection{Semantics}

We will make use of a two-dimensional semantics very similar to the one described in [26]. While the notions of a frame and model are standard, the definitions of truth and validity are modified slightly, and require that formulas be evaluated with respect to pairs of worlds: a reference index and an evaluation index. The reference index keeps track of the world currently considered actual, while it is with respect to the evaluation index that all "non-actual" elements in a formula are evaluated.

Definition 2.1 (Relational Frame). A relational frame, $\mathcal{F}$, is an ordered pair $\langle W, R\rangle$ where $W$ is a (non-empty) set and $R$ is a binary accessibility relation on $W$, i.e. $R \subseteq W \times W$.

Definition 2.2 (Relational Model). A relational model, $\mathcal{M}=\langle W, R, V\rangle$, based on a frame $\mathcal{F}$, is a triple where $V: \Phi \rightarrow \wp(W)$ is a valuation function assigning sets of possible worlds to propositional variables.

We start by considering the actuality operator in isolation. Truth is defined recursively:

$$
\begin{array}{lll}
\mathcal{M}, w_{0}, w_{1} \models p & \text { iff } & w_{1} \in V(p) \\
\mathcal{M}, w_{0}, w_{1} \models \neg \varphi & \text { iff } & \mathcal{M}, w_{0}, w_{1} \neq \varphi \\
\mathcal{M}, w_{0}, w_{1} \models \varphi \wedge \psi & \text { iff } & \mathcal{M}, w_{0}, w_{1}=\varphi \text { and } \mathcal{M}, w_{0}, w_{1} \models \psi \\
\mathcal{M}, w_{0}, w_{1} \models \varphi \vee \psi & \text { iff } & \mathcal{M}, w_{0}, w_{1}=\varphi \text { or } \mathcal{M}, w_{0}, w_{1} \models \psi \\
\mathcal{M}, w_{0}, w_{1} \models \varphi \rightarrow \psi & \text { iff } & \mathcal{M}, w_{0}, w_{1} \neq \varphi \text { or } \mathcal{M}, w_{0}, w_{1} \models \psi \\
\mathcal{M}, w_{0}, w_{1} \models \square \varphi & \text { iff } & \text { for every } w \in W, \text { if } w_{1} R w \text { then } \mathcal{M}, w_{0}, w \models \varphi \\
\mathcal{M}, w_{0}, w_{1} \models \nabla \varphi & \text { iff } & \text { there exists a } w \in W \text { s.t. } w_{1} R w \text { and } \mathcal{M}, w_{0}, w \models \varphi \\
\mathcal{M}, w_{0}, w_{1} \models @ \varphi & \text { iff } & \mathcal{M}, w_{0}, w_{0}=\varphi
\end{array}
$$

Definition 2.3 (General Satisfaction and Validity). A formula $\varphi$ is generally satisfied in a model $\mathcal{M}$ just in case, for all $w_{0}, w_{1} \in W, \mathcal{M}, w_{0}, w_{1} \models \varphi$. A formula is generally valid with respect to a class of frames iff it is generally satisfied in every model based on a frame in that class. A formula is generally valid just in case it is generally satisfied in all models.

Definition 2.4 (Diagonal Satisfaction). A formula $\varphi$ is diagonally satisfied in a model $\mathcal{M}$ just in case, for all $w \in W, \mathcal{M}, w, w \models \varphi$. A formula is diagonally valid with respect to a class of frames iff it is diagonally satisfied in every model based on a frame in that class. A formula is diagonally valid just in case it is diagonally satisfied in all models.

Australasian Journal of Logic (13:7) 2016, Article no. 1 
(It is worth explicitly pointing out that what we call "diagonal validity" is referred to as "real-world" validity in other places in the literature.)

\section{Two-Dimensional Tableaux for @}

We will describe two different basic actuality logics, corresponding to the two notions of validity introduced above. However, the two logics will not be obtained by stipulating two different systems of tableau rules. Instead, the difference between the two logics will lie in what we consider to be a proof within our tableau system. One notion of proof will end up corresponding to general validity, while the other will be the appropriate notion for diagonal validity.

Definition 3.1 (Index, Prefix, Prefixed Formula). An index is a finite sequence of natural numbers. For readability, numbers will be separated with a period. A prefix is a pair of indices separated by a comma. A prefixed formula is a wff of $\mathcal{L}+@$ preceded by a prefix. The two are separated by means of a double colon.

Intuitively, indices represent worlds, and the periods in the indices allow us to keep track of the intended accessibility between worlds. For example, $\nu . n$ represents a world accessible from $\nu$ (i.e. we should have $\nu R \nu . n$ in any corresponding frame), and so if $\varphi$ is not true at $\nu . n$, it should not be the case that $\square \varphi$ is true at $\nu$.

The doubly-indexed rules for the basic system, what we will call $\mathbf{K}+\mathbf{A}$, are:

(Non-Branching Propositional Rules) For any prefixes $\mu, \nu$ :

$$
\begin{array}{ccc}
\mu, \nu:: \varphi \wedge \psi & \mu, \nu:: \neg(\varphi \vee \psi) & \mu, \nu:: \neg(\varphi \rightarrow \psi) \\
\cline { 2 - 3 } & \frac{\mu, \nu:: \neg \varphi}{\mu, \nu: \nu} & \mu,: \varphi \\
\mu, \nu:: \psi & \mu, \nu:: \neg \psi & \mu, \nu \psi
\end{array}
$$

(Branching Propositional Rules) For any $\mu, \nu$ :

$$
\frac{\mu, \nu:: \varphi \vee \psi}{\mu, \nu:: \varphi \mid \mu, \nu:: \psi} \quad \frac{\mu, \nu:: \neg(\varphi \wedge \psi)}{\mu, \nu:: \neg \varphi \mid \mu, \nu:: \neg \psi} \quad \frac{\mu, \nu:: \varphi \rightarrow \psi}{\mu, \nu:: \neg \varphi \mid \mu, \nu:: \psi}
$$

(Double Negation) For any $\mu, \nu$ :

$$
\frac{\mu, \nu:: \neg \neg \varphi}{\mu, \nu:: \varphi}
$$

Australasian Journal of Logic (13:7) 2016, Article no. 1 
(Introductory Modal Rules) Where $\nu \cdot n$ is new to the branch:

$$
\frac{\mu, \nu:: \diamond \varphi}{\mu, \nu . n:: \varphi} \quad \frac{\mu, \nu:: \neg \square \varphi}{\mu, \nu \cdot n:: \neg \varphi}
$$

(Non-Introductory Modal Rules) Where $\nu . n$ already occurs in the branch:

$$
\frac{\mu, \nu:: \square \varphi}{\mu, \nu . n:: \varphi} \quad \frac{\mu, \nu:: \neg \nabla \varphi}{\mu, \nu . n:: \neg \varphi}
$$

And, to govern @, for any $\mu, \nu$ :

$$
\frac{\mu, \nu:: @ \varphi}{\mu, \mu:: \varphi} \quad \frac{\mu, \nu:: \neg @ \varphi}{\mu, \mu:: \neg \varphi}
$$

Again, we will call this basic system $\mathbf{K}+\mathbf{A}$. This will be the basis of all the systems we consider.

If one were interested in extending the modal basis of this system (for example to obtain $\mathbf{T}, \mathbf{D}, \mathbf{B}, \mathbf{S} \mathbf{4}, \mathbf{S 5}$, etc.), additional rules would have to be added. The obvious starting points would incorporate various of the following (here, we follow the presentation of [14, Section 6.2] and [15, Chapter $2.3])$ :

Where $\nu . n$ already occurs in the branch:

$$
\begin{aligned}
& T \quad \frac{\mu, \nu:: \square \varphi}{\mu, \nu:: \varphi} \quad \frac{\mu, \nu:: \neg \nabla \varphi}{\mu, \nu:: \neg \varphi} \\
& D \quad \frac{\mu, \nu:: \square \varphi}{\mu, \nu:: \diamond \varphi} \quad \frac{\mu, \nu:: \neg \nabla \varphi}{\mu, \nu:: \neg \square \varphi} \\
& B \quad \frac{\mu, \nu . n:: \square \varphi}{\mu, \nu:: \varphi} \quad \frac{\mu, \nu . n:: \neg \nabla \varphi}{\mu, \nu:: \neg \varphi} \\
& 4 \quad \frac{\mu, \nu:: \square \varphi}{\mu, \nu . n:: \square \varphi} \quad \frac{\mu, \nu:: \neg \nabla \varphi}{\mu, \nu . n:: \neg \nabla \varphi} \\
& 4 r \quad \frac{\mu, \nu . n:: \square \varphi}{\mu, \nu:: \square \varphi} \quad \frac{\mu, \nu . n:: \neg \nabla \varphi}{\mu, \nu:: \neg \nabla \varphi}
\end{aligned}
$$

Again, for simplicity's sake, we will restrict our attention here to logics having $\mathbf{K}$ as the modal basis. As is usual when working with modal tableaux, when one migrates to logics above $\mathbf{K}$ additional care needs to be taken at various stages of the standard proofs. This is especially true when the 
accessibility relation becomes transitive. For details on these difficulties, one can consult [12].

Throughout this paper we will assume at least some prior familiarity with the method of semantic tableaux. As such, we will not give a fully formal definition for tableaux, and will allow ourselves the luxury of relying on common intuitions. However, by way of trying to slightly more precise, we can say that for our purposes a (prefixed) tableau for a finite set of prefixed formulas $S=\left\{\alpha_{1}, \ldots, \alpha_{n}\right\}$ is an upside-down tree (where the root of the tree consists of the formulas in $S$ ) that can be defined recursively:

- The root of the tree, i.e. the prefixed formulas in $S$, written vertically with one formula per line, is a tableau;

- if $\mathcal{B}$ is a branch of a tableau $\mathcal{T}$ (i.e. a path downwards, originating in the root), then the tableau $\mathcal{T}^{\prime}$ that is obtained by extending $\mathcal{B}$ by way of appropriately applying a tableau rule to one of the formulas on $\mathcal{B}$, is also a tableau.

More casually stated, a tableau is the result of the repeated application of any of the tableau rules to the original formulas in $S$.

Definition 3.2 (Closed Tableau). A tableau branch is closed just in case it contains both $\mu, \nu:: \varphi$ and $\mu, \nu:: \neg \varphi$ for some formula $\varphi$ and prefix $\mu, \nu$. A tableau is closed if every branch of the tableau is closed.

Definition 3.3 (K $+\mathbf{A}_{\mathbf{1}}$ Tableau Proof). A closed $\mathbf{K}+\mathbf{A}$ tableau for 0,1 :: $\neg \varphi$ (i.e. where $0,1:: \neg \varphi$ is the root node), is a $\mathbf{K}+\mathbf{A}_{\mathbf{1}}$ tableau proof for $\varphi$. Any formula that has a $\mathbf{K}+\mathbf{A}_{\mathbf{1}}$ tableau proof is a $\mathbf{K}+\mathbf{A}_{\mathbf{1}}$ tableau theorem. The set of all such theorems will also be referred to by $\mathbf{K}+\mathbf{A}_{\mathbf{1}}$.

Definition 3.4 (K $+\mathbf{A}_{\mathbf{2}}$ Tableau Proof). A closed tableau for $0,0:: \neg \varphi$ (i.e. where $0,0:: \neg \varphi$ is the root node), is a $\mathbf{K}+\mathbf{A}_{\mathbf{2}}$ tableau proof for $\varphi$. Any formula that has a $\mathbf{K}+\mathbf{A}_{\mathbf{2}}$ tableau proof is a $\mathbf{K}+\mathbf{A}_{\mathbf{2}}$ tableau theorem. The set of all such theorems will also be referred to by $\mathbf{K}+\mathbf{A}_{\mathbf{1}}$.

To illustrate how a typical tableau proof might proceed, we can consider the following example.

Example 3.5. @ $\varphi \rightarrow(\diamond(@ \varphi \rightarrow \psi) \rightarrow \diamond \psi)$ is a $\mathbf{K}+\mathbf{A}_{\mathbf{1}}$ theorem. 


$$
\begin{aligned}
& 0,1:: \neg(@ \varphi \rightarrow(\diamond(@ \varphi \rightarrow \psi) \rightarrow \diamond \psi)) \\
& 0,1:: @ \varphi \\
& 0,1:: \neg(\diamond(@ \varphi \rightarrow \psi) \rightarrow \diamond \psi) \\
& 0,1:: \diamond(@ \varphi \rightarrow \psi) \\
& 0,1:: \neg \diamond \psi \\
& 0,1.2:: @ \varphi \rightarrow \psi \\
& 0,1.2:: \neg \psi \\
& 0,0:: \varphi \\
& 0,1.2:: \neg @ \varphi \quad 0,1.2:: \psi \\
& 0,0:: \neg \varphi \quad \times
\end{aligned}
$$

Unlike in the standard, single-indexed, modal case where $\mu:: \varphi$ and $\mu:: \neg \varphi$ would suffice for closure of a branch, both indices in the prefix are now relevant. For example, we would not want to close a branch on which $\mu, \nu:: @ p$ and $\sigma, \nu:: \neg @ p$ both occur, because the reference index is relevant.

Our definitions, in light of the tableau rules above, do allow for a particular oddity about which we have to take some care. Specifically, given our definitions and rules thus far, we will not be able to close certain tableaux that, in general, represent contradictory states of affairs. For example, the occurrence of $0,1:: p$ and $2,1:: \neg p$ on a branch will not suffice to close that branch, even though it represents a contradictory state of affairs.

There are two possible solutions to this issue, and we will adopt each of them in different parts of the paper.

The first option is to just ignore the problem, and to notice that when one is actually trying to construct a proof of a formula, the above situation cannot arise.

Observation 3.6. Let $i$ be 1 or 2 . Then, all prefixed formulas of any $\mathbf{K}+\mathbf{A}_{\mathbf{i}}$ tableau starting from $0, \nu:: \alpha$ have a prefix of the form $0, \mu$.

This is because, while our definition of tableaux allows for multiple formulas to occupy the root, our notion of proof does not, and we always root a tableau proof with a single formula, rather than a set. In particular, we always start with something of the form $0,1:: \alpha$ (or $0,0:: \alpha$ in the case of $\mathbf{K}+\mathbf{A}_{\mathbf{2}}$ ), and, when our operators are restricted to just @ and the modalities, there is no way of introducing, via our rules, a new index in the first prefix position. Thus, as a practical matter, the problematic cases will not 
arise; our notion of provability is unaffected by the lack of closure in these circumstances.

While in practice these cases do not occur, that does not mean they will not arise during our completeness construction. And, in fact, they do. However, we can overcome these difficulties by restricting our truth lemma slightly, and focusing on particular prefixes. This solution, however, has both a payoff and a cost: the resulting truth lemma is less general than might be hoped, but we need not amend our tableau rules.

However, this approach is not available once we enrich our languages with other operators allowing for greater flexibility with respect to the reference index. This is the case with both of the other operators we will consider in this paper: the fixedly operator $F$ and the Vlach-operator $\uparrow$. In those cases, we need to alter the proof system slightly to ensure the completeness construction works as desired. Details of this are below.

Finally, it is worth explicitly pointing out that one could add to the system $\mathbf{K}+\mathbf{A}$ a rule governing this problematic scenario (as we will with the other operators) without encountering any problems (all that is required is an extra case in a couple of proofs). Indeed, in some way this alternative system is more general. We will not adopt this method immediately, however, as we find it interesting, if only by way of comparison, to consider both approaches.

\subsection{Soundness}

Definition 3.7 (Tableau Satisfiability). Let Gamma be a set of prefixed formulas, and let $\mathcal{I}$ be the set of all indices occurring in $\Gamma$. We say that $\Gamma$ is satisfiable in a model $\mathcal{M}=\langle W, R, V\rangle$ just in case there is a function, $\theta: \mathcal{I} \rightarrow W$, s.t.

1. If $\mu$ and $\mu . n$ both occur as indices in $\Gamma$, then $\theta(\mu) R \theta(\mu . n)$.

2. If $\mu, \nu:: \varphi \in \Gamma$, then $\mathcal{M}, \theta(\mu), \theta(\nu) \models \varphi$.

When such a model and function exist, we can just say that $\Gamma$ is satisfiable. A branch of a tableau is satisfiable when the set of formulas on the branch are satisfiable. A tableau is satisfiable if one of its branches is satisfiable.

Full proofs for the following results, for the standard modal cases, can be found in [15, Section 2.5].

Observation 3.8. A closed tableau is not satisfiable. 
Proof. Straightforward. For detail, see [15].

Observation 3.9. Given a satisfiable tableau, the tableau remains satisfiable after the application of one of the above tableau rules.

Proof. We only consider the cases for @ rules here.

Letting $\mathcal{T}$ be a tableau, and $\mathcal{B}$ a branch of $\mathcal{T}$, assume that $\mu, \nu:: @ \varphi \in \mathcal{B}$ and that $\mathcal{B}$ is satisfiable, i.e., $\mathcal{M}, \theta(\mu), \theta(\nu) \models @ \varphi$. Then $\mathcal{M}, \theta(\mu), \theta(\mu) \models \varphi$ and so $\mathcal{B} \cup\{\mu, \mu:: \varphi\}$ is satisfiable using $\theta$.

Lastly, assume $\mu, \nu:: \neg @ \varphi \in \mathcal{B}$ and that $\mathcal{B}$ is satisfiable. So $\mathcal{M}, \theta(\mu), \theta(\nu) \models$ $\neg @ \varphi$, which just means $\mathcal{M}, \theta(\mu), \theta(\nu) \not=@ \varphi$. This holds iff $\mathcal{M}, \theta(\mu), \theta(\mu) \not \models$ $\varphi$ which is equivalent to $\mathcal{M}, \theta(\mu), \theta(\mu) \models \neg \varphi$. Thus $\mathcal{B} \cup\{\mu, \mu:: \neg \varphi\}$ is satisfiable.

Note that this theorem is general in the sense that it applies in case $\mu=\nu$ or $\mu \neq \nu$.

Theorem 3.10. If $\varphi$ has an $\mathbf{K}+\mathbf{A}_{\mathbf{1}}$ tableau proof, it is generally valid.

Proof. Suppose, for contradiction, that $\varphi$ has a tableau proof, but is not generally valid. Then there exists a closed tableau, $\mathcal{T}$, beginning with $0,1:$ : $\neg \varphi$. Let $\mathcal{T}_{0}=\{0,1:: \neg \varphi\}$. Furthermore, there must be some model $\mathcal{M}$, containing worlds $w_{0}$ and $w_{1}$ s.t. $\mathcal{M}, w_{0}, w_{1} \not \models \varphi$. Letting $\theta(0)=w_{0}$ and $\theta(1)=w_{1}$, we see that $\mathcal{T}_{0}$ is satisfiable. Therefore, from Observation $3.9, \mathcal{T}$ must be satisfiable. But we know that closed tableau cannot be satisfied, so there is a contradiction.

Theorem 3.11. If $\varphi$ has an $\mathbf{K}+\mathbf{A}_{\mathbf{2}}$ tableau proof, it is diagonally valid.

Proof. Suppose, for contradiction, that $\varphi$ has a tableau proof, but is not valid. Then there exists a closed tableau, $\mathcal{T}$, beginning with $0,0:: \neg \varphi$. Let $\mathcal{T}_{0}=\{0,0:: \neg \varphi\}$. Furthermore, there must be some model $\mathcal{M}$, containing a world $w_{0}$ s.t. $\mathcal{M}, w_{0}, w_{0} \not \models \varphi$. Letting $\theta(0)=w_{0}$, we see that $\mathcal{T}_{0}$ is satisfiable. Therefore, from Observation $3.9, \mathcal{T}$ must be satisfiable. Again, this is a contradiction

The above results establish tableau general soundness for $\mathbf{K}+\mathbf{A}_{\mathbf{1}}$ and diagonal soundness for $\mathbf{K}+\mathbf{A}_{\mathbf{2}}$.

\subsection{Completeness}

Our approach to completeness is that of [14, Section 6]. We will omit many details in our proofs that can be found there. 
Definition 3.12 ( $\mathbf{K}+\mathbf{A}$-consistent). A set $\Gamma$ of prefixed formulas is said to be $\mathbf{K}+\mathbf{A}$-consistent if no $\mathbf{K}+\mathbf{A}$ tableau for a finite subset of $\Gamma$ is closed (where the finite subset is the "root" of the tree).

We will call $\Gamma$ maximally consistent when it is consistent, and the addition of any further prefixed formula would result in an inconsistency.

However, as mentioned before, we must be careful. Specifically, this definition results in $\{0,1:: p, 2,1:: \neg p\}$ being considered $\mathbf{K}+\mathbf{A}$-consistent. Thus, when we actually make use of this definition, we will have to ensure that our $\mathbf{K}+\mathbf{A}$-consistent sets obey additional constraints as well.

Definition 3.13 ( $\pi$-complete). A formula is a $\pi$-formula if it is of the form $\diamond \varphi$ or $\neg \square \varphi$. If $\pi$ is $\diamond \varphi$, let $\pi_{0}$ be $\varphi$. If $\pi$ is $\neg \square \varphi$, let $\pi_{0}$ be $\neg \varphi$. A set $\Gamma$ of prefixed formulas is said to be $\pi$-complete when:

$$
\text { if } \mu, \nu:: \pi \in \Gamma \text {, then } \mu, \nu . k:: \pi_{0} \in \Gamma \text { for some } k \in \mathbb{N} \text {. }
$$

Definition 3.14 (Omits Infinitely Many Integers). A set of prefixed formulas $\Gamma$ omits infinitely many integers just in case the size of the set of positive integers not appearing in prefixes of $\Gamma$ is infinite.

Lemma 3.15 (Lindenbaum-Henkin Construction). If $\Gamma$ is an $\mathbf{K}+\mathbf{A}$-consistent set of prefixed sentences that omits infinitely many integers, then there is a maximally $\mathbf{K}+\mathbf{A}$-consistent set of prefixed formulas, $K$, such that $\Gamma \subseteq K$. Furthermore, $K$ is $\pi$-complete.

Proof. Suppose $\Gamma$ is an $\mathbf{K}+\mathbf{A}$-consistent set of prefixed sentences that omits infinitely many integers. Enumerate all prefixed formulas in the language ${ }^{5}$ : $\mu_{1}, \nu_{1}:: \alpha_{1}, \mu_{2}, \nu_{2}:: \alpha_{2}, \ldots$ Define the following sequence of sets:

$\Gamma_{1}=\Gamma$

If $\alpha_{n}$ is not a $\pi$-formula, and if $\Gamma_{n} \cup\left\{\mu_{n}, \nu_{n}:: \alpha_{n}\right\}$ is $\mathbf{K}+\mathbf{A}$-consistent, then let $\Gamma_{n+1}=\Gamma_{n} \cup\left\{\mu_{n}, \nu_{n}:: \alpha_{n}\right\}$.

If $\alpha_{n}$ is a $\pi$-formula, and if $\Gamma_{n} \cup\left\{\mu_{n}, \nu_{n}:: \alpha_{n}\right\}$ is $\mathbf{K}+\mathbf{A}$-consistent, then let $\Gamma_{n+1}=\Gamma_{n} \cup\left\{\mu_{n}, \nu_{n}:: \pi, \mu_{n}, \nu_{n} . k:: \pi_{0}\right\}$ where $\nu . k$ does not occur in $\Gamma_{n}$. (Note that such $k$ will always be available since $\Gamma_{n}$ will always omit infinitely many integers. Also, if $\Gamma_{n} \cup\left\{\mu_{n}, \nu_{n}:: \pi\right\}$ is consistent, then $\Gamma_{n} \cup\left\{\mu_{n}, \nu_{n}:: \pi\right.$, $\left.\mu_{n}, \nu_{n} . k:: \pi_{0}\right\}$ will be as well, so long as $k$ is new.)

Finally, if neither of these situations obtain, take $\Gamma_{n+1}=\Gamma_{n}$.

\footnotetext{
${ }^{5}$ Note that the set of all double-indexed formulas is countable: the set of all indices is $\mathbb{N}^{<\infty}$ - the set of all finite sequences of natural numbers - and so the set of all possible double-indexed formulas has size $\left|\mathbb{N}^{<\infty}\right| \times|\mathbb{N}<\infty| \times \mid$ Form $\mid$, which is countable since $\mathbb{N}<\infty$ and Form both are (where Form is the set of wff).
}

Australasian Journal of Logic (13:7) 2016, Article no. 1 
Let $K=\bigcup_{n \in \omega} \Gamma_{n}$.

That $K$ is $\pi$-complete is obvious from the construction. If $\mu_{n}, \nu_{n}:: \pi$ was added at the $n^{t h}$ stage, then so was $\mu_{n}, \nu_{n} . k:: \pi_{0}$. Furthermore, since the $k$ is new (the availability of which is guaranteed since $\Gamma_{n}$ omits infinitely many integers), if $\Gamma_{n} \cup\{\mu, \nu:: \pi\}$ is consistent, then $\Gamma_{n} \cup\left\{\mu, \nu . k:: \pi_{0}\right\}$ will also be.

It is equally straightforward that $K$ is $\mathbf{K}+\mathbf{A}$-consistent, since it remains so at each stage of the construction by definition. If it were not consistent, there would be a finite subset $\Gamma^{\prime}$, which has a closed tableau. But all the elements of $\Gamma^{\prime}$ must have appeared in the enumeration by some point, $n$. Thus, we would have that $\Gamma_{n}$ is inconsistent, which is impossible.

Thus, it remains only to be shown that $K$ is maximal in the sense that we cannot add any new prefixed formula without creating an inconsistency. Assume this to be false. That is, assume there is some prefixed formula $\mu, \nu:: \alpha$ that can be added without creating an inconsistency. But this formula must have appeared in our enumeration at some stage. As such, if adding it would not have resulted in an inconsistency, it would have been added at that stage.

Note, also, that $K$ is saturated. That is, for a given prefixed formula $\mu, \nu:: \varphi \in K$, the prefixed formulas (or one of them, in the case of the disjunctive rules) arising from the application of the relevant tableau rule must also be elements of $K$.

We are now in a position to demonstrate that a suitable model satisfying any non-theorem of $\mathbf{K}+\mathbf{A}_{\mathbf{1}}$ can be found. If $\varphi$ is not $\mathbf{K}+\mathbf{A}_{\mathbf{1}}$-provable using the tableau rules, then, by definition, $\{0,1:: \neg \varphi\}$ is $\mathbf{K}+\mathbf{A}$ consistent. Obviously this satisfies the criteria of the above construction, so we can extend to a maximally consistent and $\pi$-complete set $S$. Let $W$ be the set of indices occurring in $S$. Define $R$ over the set $W$ s.t. for $\mu, \nu \in W$, $\mu R \nu$ iff $\nu=\mu . n$ for some $n \in \mathbb{N}$. Finally, define the valuation $V$ by letting $\nu \in V(p)$ iff $0, \nu:: p \in S$. (This restricted definition of $V$, and a more specific statement of the following truth lemma, is what allows us to circumvent the problem presented by the potential appearance of both $\mu, \nu:: p$ and $\sigma, \nu:: \neg p$.)

Finally, let $\mathcal{M}=\langle W, R, V\rangle$.

Lemma 3.16. For every formula $\alpha$, and every index $\nu$,

$$
\text { if } 0, \nu:: \alpha \in S \text {, then } \mathcal{M}, 0, \nu \mid=\alpha \text {. }
$$

Australasian Journal of Logic (13:7) 2016, Article no. 1 


$$
\text { if } 0, \nu:: \neg \alpha \in S \text {, then } \mathcal{M}, 0, \nu \not \neq \alpha \text {. }
$$

Proof. Given Lemma 3.15, this result proceeds by (a very long, but straightforward) induction on the complexity of formulas. We will omit everything except the base cases and the cases concerning @.

We have two base cases: $\alpha$ is a propositional variable, and $\alpha$ is the negation of a propositional variable.

If $0, \nu:: p \in S$, then, by definition of $\mathcal{M}, \nu \in V(p)$. So $\mathcal{M}, 0, \nu \models p$.

If $0, \nu:: \neg p \in S$, then, since $S$ is consistent, we know that $0, \nu:: p \notin S$. This means that $\nu \notin V(p)$, and so $\mathcal{M}, 0, \nu \not \models p$.

We now consider the two @ cases.

Let $\alpha$ be of the form @ $\varphi$ and take $0, \nu:: @ \varphi \in S$. Thus, $0,0:: \varphi \in S$. Then, by the induction hypothesis, $\mathcal{M}, 0,0 \models \varphi$, which means $\mathcal{M}, 0, \nu \models @ \varphi$.

Assume $\alpha$ is of the form $\neg @ \varphi$. If $0, \nu:: \neg @ \varphi \in S$ then $0,0:: \neg \varphi \in S$. Then, by the induction hypothesis, $\mathcal{M}, 0,0 \not \models \varphi$, which means $\mathcal{M}, 0, \nu \not \models @ \varphi$.

Corollary $\mathbf{3 . 1 7}$ (Tableau Completeness for $\mathbf{K}+\mathbf{A}_{\mathbf{1}}$ ). If $\alpha$ is generally valid, it has a $\mathbf{K}+\mathbf{A}_{\mathbf{1}}$ tableau proof.

Corollary 3.18 (Tableau Completeness for $\mathbf{K}+\mathbf{A}_{\mathbf{2}}$ ). If $\alpha$ is diagonally valid, it has a $\mathbf{K}+\mathbf{A}_{\mathbf{2}}$ tableau proof.

Proof. We prove the contrapositive. That is, assume $\varphi$ does not have a $\mathbf{K}+\mathbf{A}_{\mathbf{2}}$ tableau proof. By definition, this means that the tableau rooted with $0,0:: \neg \varphi$ does not close, and is therefore $\mathbf{K}+\mathbf{A}$-consistent. Thus, as before, we can extend $\{0,0:: \neg \varphi\}$ to a maximally consistent, $\pi$-complete set $S$. Defining $\mathcal{M}=\langle W, R, V\rangle$ as before, we can utilize the same truth lemma as for $\mathbf{K}+\mathbf{A}_{\mathbf{1}}$ to show that there must be a model s.t. $\mathcal{M}, 0,0 \not \models \varphi$. Thus, $\varphi$ must not be diagonally valid.

\section{Decidability}

In this section we present a systematic tableau proof procedure for our actuality logics. Though one could also prove completeness via this method, we will refrain from doing so, and refer interested readers to [13] or [18]. Our approach is that of [13], Chapter 8, and [18], and their proofs go through largely unaltered, as the presence of a second index in the prefix turns out to require only minor alterations. Because @ is eliminable in the basic propositional modal language with actuality, the primary advantage of @ in these settings is one of transparency: some things are just easier (at 
least in the sense of being more intuitive) to formalize, or express, with @ than without it. However, were a decidability result not straightforwardly forthcoming for the tableaux, the price of such expressive convenience, given its lack of necessity, would not likely be worth the cost. Thus, the results in this section are intended to be understood not so much as some great feature of the logic, but rather as demonstrating that the systems adhere to a kind of minimal requirement. ${ }^{6}$ In addition, decidability proofs for logics with @ have been offered before, for example in [20].

We deal with modal formulas in the manner described in [18]. In particular, we employ the following terminology: in the course of constructing a tableau, a formula is awake if the appropriate tableau rules have not yet been applied to it; a formula is finished if the appropriate tableau rules have been applied to it and it will not need to be considered again; a formula is asleep if the appropriate tableau rules have been applied, but we may have to consider the formula again if new indices are added to the branches on which it occurs. ${ }^{7}$

Given a formula $\varphi$ in the language of $\mathcal{L}+@$, we can construct the tableau rooted with $\chi$ as follows:

\section{Begin}

Step 1: Place $0,1:: \neg \chi$ as the root of the tableau. Mark this as awake.

While the tableau is open and there are awake formulas, do:

Step $\mathbf{n + 1}$ : In a breadth-first manner, choose the first awake prefixed formula, $\mu, \nu:: \alpha$. (That is, select the highest awake prefixed formula, and, if there are several such formulas on the same level, choose the left-most formula.) For each open branch $\mathcal{B}$ through $\mu, \nu:: \alpha$, do:

(Propositional Variable): If $\alpha$ is a propositional variable $p$, or the negation of a propositional variable, do nothing.

(Non-Branching Propositional): If $\alpha$ is of the form $\varphi \wedge \psi, \neg(\varphi \vee \psi)$, $\neg(\varphi \rightarrow \psi)$, or $\neg \neg \varphi$ apply the appropriate non-branching propositional rule, adding the relevant formula(s) to the bottom of $\mathcal{B}$, provided they do not already appear on the branch, and mark them as awake.

(Branching Propositional): If $\alpha$ is of the form $\varphi \vee \psi, \neg(\varphi \wedge \psi)$, or $\varphi \rightarrow$ $\psi$, fork $\mathcal{B}$ into two branches, $\mathcal{B}_{1}$ and $\mathcal{B}_{2}$ and add the appropriate prefixed

\footnotetext{
${ }^{6}$ Decidability for extensions containing $F$ and $\uparrow$ is discussed in the appendix.

${ }^{7}$ We remain focused on actuality logics having $\mathbf{K}$ as their modal basis, and so do not consider possible extensions here. If one wished to consider such avenues, the usual complications concerning cycle checking would have to be confronted (for how these issues are handled normally, one might consult [13] or [18]).
}

Australasian Journal of Logic (13:7) 2016, Article no. 1 
formula (as dictated by the above rules) to the end of each branch, so long as it doesn't already appear on the branch, and mark it as awake. (E.g. in the case of $\varphi \vee \psi$, add $\mu, \nu:: \varphi$ to $\mathcal{B}_{1}$ and $\mu, \nu:: \psi$ to $\mathcal{B}_{2}$.)

(Introductory Modal Rules): If $\alpha$ is a $\pi$-formula (i.e. of the form $\nabla \varphi$ or $\neg \square \varphi$ ), add $\mu, \nu . n:: \pi_{0}$ to the bottom of $\mathcal{B}$ where $n$ is the least natural number such that $n$ is new to $\mathcal{B}$. Mark $\mu, \nu . n:: \pi_{0}$ as awake. Furthermore, mark each formula of the form $\sigma, \nu:: \square \psi$ or $\sigma, \nu:: \neg \nabla \psi$ on $\mathcal{B}$ as awake.

(Non-Introductory Modal Rules): If $\alpha$ is of the form $\neg \nabla \varphi$ or $\square \varphi$, add to the bottom of $\mathcal{B} \mu, \nu . n:: \neg \varphi$ or $\mu, \nu . n:: \varphi$, respectively, for each $\nu . n$ occuring on $\mathcal{B}$, provided $\mu, \nu . n:: \varphi$ does not already appear on $\mathcal{B}$, and mark each as awake.

(Actuality Rules): If $\alpha$ is of the form @ $\varphi$ or $\neg @ \varphi$, then add $\mu, \mu:: \varphi$ or $\mu, \mu:: \neg \varphi$, respectively, to the end of $\mathcal{B}$, provided it does not already appear on $\mathcal{B}$, and mark it as awake.

Once each branch through $\mu, \nu:: \alpha$ has been updated appropriately, if $\alpha$ is of the form $\square \varphi$ or $\neg \nabla \varphi$, mark $\mu, \nu:: \alpha$ as asleep. Otherwise, mark it as finished. End step $n+1$.

\section{End}

Theorem 4.1. Given a formula $\chi$ in the language $\mathcal{L}+@$, any attempt to prove $\chi$ according to the above procedure terminates in a finite number of steps.

Proof. Assume not. That is, assume that the process described above can go on forever. This involves the creation of an infinite tableau. (Note that every "awakening" is accompanied by an extension of some branch.)

So, by König's lemma, we must have an infinitely long branch $\mathcal{B}$.

Consider the set of indices occurring on $\mathcal{B}$ :

$$
\mathcal{P}=\{\mu \mid 0, \mu:: \varphi \in \mathcal{B} \text { for some } \varphi\} .
$$

For $\mathcal{B}$ to be infinite, $\mathcal{P}$ must also be infinite. For if we take $\mathcal{P}$ to be finite, then the number of prefixes in $\mathcal{B}$ is bounded by $|\{0\} \times \mathcal{P}|$. Also, taking $\operatorname{SubForm}(\chi)$ to be the (finite) set consisting of all subformulas of $\chi$ along with the negation of all such formulas, the set of all possible formulas on $\mathcal{B}$ is $(\{0\} \times \mathcal{P}) \times S u b F$ orm $(\chi)$, which is finite. Therefore, the only way we could have an infinite $\mathcal{B}$ with a finite $\mathcal{P}$ would be if one of these prefixes occurs an infinite number of times. However, this is not possible. For any prefixed formula $\mu, \nu:: \varphi, \varphi$ must either be a subformula of $\chi$ or the negation of such a subformula. As there are only finitely many such formulas, and 
we avoid adding multiple occurrences of any particular prefixed formula, we can only have a finite number of formulas prefixed by a particular pair on $\mathcal{B}$.

Given an index $\mu$, define the length of $\mu$, denoted length $(\mu)$, to the be one more than the number of separating '.' that occur in the index. That is, the length of the index ' 1.1 ' is two. The length of '1.2.1' is three, etc.

For each $n \in \mathbb{N}$ greater than 0 , define

$$
\mathcal{P}_{n}=\{\mu \mid \mu \in \mathcal{P} \text { and length }(\mu)=n\} .
$$

Then $\mathcal{P}=\bigcup_{1 \leq n \in \mathbb{N}} \mathcal{P}_{n}$

There are now two possibilities allowing $\mathcal{P}$ to be infinite.

First, there is some $n \in \mathbb{N}$ such that $\left|\mathcal{P}_{n}\right|=\omega$. Alternatively, there are infinitely many $n$ such that $\mathcal{P}_{n} \neq \emptyset$.

We consider these possibilities in turn.

In the first case, let $n \in \mathbb{N}$ be the smallest number such that $\left|\mathcal{P}_{n}\right|=\omega$. Notice, however, that for there to be an infinite number of indices of length $n$ (for $n>1$ ) there must be an infinite number of indices with length $n-1$. This is because new indices of length $n$ (for $n>1$ ) can only be attached to the branch via the application of a $\pi$-rule to a prefixed $\pi$-formula where the second index is of length $n-1$, and $\pi$ formulas are marked as "finished" as soon as they are dealt with. Furthermore, as argued above, each numeral can appear as the second index only a finite number of times. Thus, for there to be an $n$ s.t. $\left|\mathcal{P}_{n}\right|=\omega$, we would have to have an infinite number of indices with length 1 . However, the only two indices of length 1 that occur in our prefixes are 0 and 1 , and each of these can only occur a finite number of times. Therefore, this case is impossible.

Moving to the second possibility then, assume that there are infinitely many $n$ such that $\mathcal{P}_{n} \neq \emptyset$. Let $m$ be the modal degree of $\chi$ (i.e. the number of modal operators occuring in $\chi$ ). Then the maximum length of the second index in the prefix is $m+1$, since indices can only be lengthed by applying the relevant $\pi$-rules. This, however, contradicts the possiblity of there being infinitely many $n$ such that $\mathcal{P}_{n} \neq \emptyset$.

\section{Tableaux for the Fixedly Operator}

In [10], Martin Davies and Lloyd Humberstone, as a means of capturing the intuition that being an actual world is a contingent property, address 
the benefits of including in their actuality logic an operator, F, called the 'Fixedly' operator, allowing one to quantify over actual worlds. Davies, in [9], motivates such an operator in the following manner:

The semantic rule for the 'Actually'-operator, 'A', has the result that if 'As' is true with respect to any world then it is true with respect to every world. So if 'As' is true then so is ' $\square$ As'. While this is an immediate consequence of the intuitive semantics for ' $A$ ', it does not accord well with the idea that it is a largely contingent matter what is actually the case. Suppose, for example, that the embedded sentence $s$ means that the earth moves, and that this is contingently true. Then, even allowing that there is a notion of necessity expressed by the modal operator ' $\square$ ' on which 'As' is necessarily true (that is, ' $\square$ As' is true), we also want to say that there is another notion of necessity on which 'As' is not necessarily true. This second notion of necessity is needed to capture the intution that it is a contingent matter which possible world is actual. ${ }^{8}$ (pp. 85)

In other words, and in our notation, while @ $p$ implies its necessitation, $\square @ p$, we would like to be able to articulate the fact that $p$ 's actuality is, or might be, a contingent matter - contingent upon which world we take as the actual one. The fixedly operator allows us to say just this. For example, @ $p \wedge \neg \mathrm{F} @ p$ tells us that while $p$ is true in the actual world, there is some other world such that, were we to consider it actual, @p would no longer hold.

This all motivates the following semantic clause for the fixedly operator ${ }^{9}$ :

$$
\mathcal{M}, w_{0}, w_{1} \models \mathrm{F} \varphi \text { iff } \mathcal{M}, w, w_{1} \models \varphi \text { for all } w \in W
$$

As Davies [9] points out, it is not the fixedly operator in isolation that allows us to capture the desired second notion of necessity; if $\varphi$ contains no occurences of @, then $\mathrm{F} \varphi$ is true if and only if $\varphi$ is. Rather, it is the fixedly operator combined with the actuality operator that gives rise to the new form of necessity and articulates the likes of "regardless of which world is actual, some proposition actually holds".

In these cases we have $\mathcal{M}, w_{0}, w_{1}=\mathrm{F} @ \varphi$

\footnotetext{
${ }^{8}$ In [9] and [10] the underlying semantics, as well as the underlying modal logic (S5), differs from ours, but the relevance of the discussion is unaffected.

${ }^{9}$ We note that the fixedly operator behaves in a very similar manner to Segerberg's $\boxminus$ [25].
}

Australasian Journal of Logic (13:7) 2016, Article no. 1 
iff $\mathcal{M}, w, w_{1}=@ \varphi$ for all $w \in W$

iff $\mathcal{M}, w, w \models \varphi$ for all $w \in W$.

Or, in other words, just in case $\varphi$ is diagonally satisfied in $\mathcal{M}$.

\subsection{Tableau Rules for $F$}

We can supplement the tableau rules of $\mathbf{K}+\mathbf{A}$ with the following two rules governing $\mathrm{F}$ :

For $\sigma$ already occuring in the branch:

$\frac{\mu, \nu:: \mathrm{F} \varphi}{\sigma, \nu:: \varphi}$

Where $\sigma$ is new on the branch:

$\frac{\mu, \nu:: \neg \mathrm{F} \varphi}{\sigma, \nu:: \neg \varphi}$

Finally, we need one more rule to ensure our trees close. Consider the formula $\neg(p \wedge \neg \mathrm{F} p)$. This is valid. ${ }^{10}$ However, it is not provable with the current set of rules. Consider the following attempted tableau proof:

$0,1:: \neg \neg(p \wedge \neg \mathbf{F} p)$

$0,1:: p \wedge \neg \mathrm{F} p$

$0,1:: p$

$0,1:: \neg \mathrm{F} p$

$2,1:: \neg p$

The problem is that we have used all available rules, but cannot close the tableau as we would like. Furthermore, we cannot simply ignore the issue and adjust our completeness proof as we did before. This is because we now have the ability to modify the first index in the prefix, and so our inability to close tableaux of this sort actually affects the formulas we classify as provable (unlike before). Also, restricting our truth lemma is not possible because of the cases for the $F$ operator. Thus, we need to add a new rule to deal explicitly with this scenario. Specifically, we add the following:

$\frac{\mu, \nu:: p}{\sigma, \nu:: p}$

Where $\sigma$ occurs on the branch.

The system $\mathbf{K}+\mathbf{A}+\mathbf{F}$ is obtained by adding these three new rules to those of $\mathbf{K}+\mathbf{A}$.

\footnotetext{
${ }^{10}$ Because $p \rightarrow \mathrm{F} p$ will be a theorem of fixedly logic, while $\varphi \rightarrow \mathrm{F} \varphi$ in general will not (when $\varphi$ contains occurrences of @), the logic of fixedly is not closed under uniform substitutions.
} 


\subsection{Soundness}

Since we are merely adding new tableau rules, we can prove soundness and completeness simply by supplementing the above proofs (from section 3 ) with the cases governing the new rules for $F$. (The notions of proof are the same as before - for example, in the presence of the fixedly operator, a $\mathbf{K}+\mathbf{A}_{\mathbf{1}}$ proof becomes a $\mathbf{K}+\mathbf{A}_{\mathbf{1}}+\mathbf{F}$ proof.)

Lemma 5.1. Given a satisfiable tableau, the tableau remains satisfiable after the application of one of the above tableau rules.

Proof. We need only add to the proof of Observation 3.9 above. So, as before, let $\mathcal{T}$ be a tableau, and $\mathcal{B}$ a satisfiable branch of $\mathcal{T}$.

When $\mathcal{B}$ contains $\mu, \nu:: \mathrm{F} \varphi$, we have that $\mathcal{M}, \theta(\mu), \theta(\nu) \models \mathrm{F} \varphi$. Thus, for all $w \in W, \mathcal{M}, w, \theta(\nu) \models \varphi$, and, in particular, for all $\sigma$ occuring on $\mathcal{B}$ we have that $\mathcal{M}, \theta(\sigma), \theta(\nu) \models \varphi$. Thus $\mathcal{B} \cup\{\sigma, \nu:: \varphi\}$ is satisfied by $\theta$.

For a $\mathcal{B}$ containing $\mu, \nu:: \neg \mathrm{F} \varphi$, we have $\mathcal{M}, \theta(\mu), \theta(\nu) \models \neg \mathrm{F} \varphi$, and so there must exist a $w \in W$ where $\mathcal{M}, w, \theta(\nu) \models \neg \varphi$. Define $\theta^{\prime}$ to be $\theta$ extended with $\theta^{\prime}(\sigma)=w$ where $\sigma$ does not yet occur on $\mathcal{B}$ (and is therefore undefined for $\theta$, ensuring that $\theta^{\prime}$ is well-defined). Thus $\mathcal{M}, \theta^{\prime}(\sigma), \theta(\nu) \models \neg \varphi$, and so $\mathcal{B} \cup\{\sigma, \nu:: \neg \varphi\}$ is satisfied by $\theta^{\prime}$.

Assume now that $\mathcal{B}$ contains $\mu, \nu:: p$ and that $\mathcal{M}, \theta(\mu), \theta(\nu) \models p$. This means that $\theta(\nu) \in V(p)$. Therefore, for any $w \in W$, we will have that $\mathcal{M}, w, \theta(\nu) \models p$. But, given that $W$ is the codomain of $\theta$, we certainly have that $\mathcal{M}, \theta(\sigma), \theta(\nu) \models p$ for any $\sigma$ occuring on $\mathcal{B}$. Thus, $\sigma, \nu:: p$ is satisfiable by $\theta$ for all such $\sigma$.

Corollary 5.2. If $\varphi$ has an $\mathbf{K}+\mathbf{A}_{\mathbf{1}}+\mathbf{F}$ tableau proof, then it is generally valid.

Corollary 5.3. If $\varphi$ has an $\mathbf{K}+\mathbf{A}_{\mathbf{2}}+\mathbf{F}$ tableau proof, then it is diagonally valid.

\subsection{Completeness}

Previously, we used the notion of $\pi$-completeness (for a set of prefixed formulas) to address the issue of the new prefixes required by possibility formulas. We need to do a similar thing now for the fixedly formulas. In particular, we want to make sure that when we perform the Lindenbaum-Henkin construction, not only do we have the necessary prefixed formulas dealing with the modalities, but also those for the fixedly formulas. 
Definition 5.4 (Prefix-Complete). A set of formulas is prefix-complete whenever:

- if $\mu, \nu:: \pi \in \Gamma$, then $\mu, \nu . k:: \pi_{0} \in \Gamma$ for some $k \in \mathbb{N}$;

- if $\mu, \nu:: \neg \mathrm{F} \varphi \in \Gamma$, then $\sigma, \nu:: \neg \varphi \in \Gamma$ for some $\sigma \in \mathbb{N}$.

(Where the definition of $\pi$-formulas given in Definition 3.13 is unchanged.)

We can now proceed with our new Lindenbaum-Henkin construction. (Our notion of consistency is now one of $\mathbf{K}+\mathbf{A}+\mathbf{F}$-consistency.)

Lemma 5.5 (Lindenbaum-Henkin Construction for Fixedly). Suppose $\Gamma$ is an $\mathbf{K}+\mathbf{A}+\mathbf{F}$-consistent set of prefixed sentences that omits infinitely many integers. Then there exists a prefix-complete and maximally consistent $K$ s.t. $\Gamma \subseteq K$.

Proof. Enumerate all prefixed formulas in the language: $\mu_{1}, \nu_{1}:: \alpha_{1}, \mu_{2}, \nu_{2}::$ $\alpha_{2}, \ldots$ Define the following sequence of sets:

$\Gamma_{1}=\Gamma$

If $\alpha_{n}$ is neither a $\pi$-formula nor a formula beginning with $\neg \mathrm{F}$, and if $\Gamma_{n} \cup\left\{\mu_{n}, \nu_{n}:: \alpha_{n}\right\}$ is consistent, then let $\Gamma_{n+1}=\Gamma_{n} \cup\left\{\mu_{n}, \nu_{n}:: \alpha_{n}\right\}$.

If $\alpha_{n}$ is a $\pi$-formula, and if $\Gamma_{n} \cup\left\{\mu_{n}, \nu_{n}:: \alpha_{n}\right\}$ is consistent, then let $\Gamma_{n+1}=\Gamma_{n} \cup\left\{\mu_{n}, \nu_{n}:: \pi, \mu_{n}, \nu_{n} . k:: \pi_{0}\right\}$ where $\nu . k$ does not occur in $\Gamma_{n}$.

If $\alpha_{n}$ is a $\neg \mathrm{F}$-formula of the form $\neg \mathrm{F} \varphi$, and if $\Gamma_{n} \cup\left\{\mu_{n}, \nu_{n}:: \alpha_{n}\right\}$ is consistent, then let $\Gamma_{n+1}=\Gamma_{n} \cup\left\{\mu_{n}, \nu_{n}:: \neg \mathbf{F} \varphi, \sigma, \nu_{n}:: \neg \varphi\right\}$ where $\sigma$ does not occur in $\Gamma_{n}$.

Finally, in the last case, if none of these prior situations obtain, take $\Gamma_{n+1}=\Gamma_{n}$.

As before, each $\Gamma_{n}$ will be consistent and omit infinitely many integers.

We can now construct a model that will satisfy a truth lemma. If $\varphi$ is not provable in $\mathbf{K}+\mathbf{A}_{\mathbf{1}}+\mathbf{F}$, then $0,1:: \neg \varphi$ is consistent. Thus, using the above Lindenbaum-Henkin construction, we can create a set $S$ that is maximally consistent and prefix-complete. From $S$, we can construct a model failing to satisfy $\varphi$ by letting $W$ be the set of indices occurring in $S, \mu R \nu$ iff $\nu=\mu$. $k$ for some $k \in \mathbb{N}$, and $\nu \in V(p)$ iff $\mu, \nu:: p \in S$ for some $\mu$ (note that this will imply it is so for any $\mu$ ).

Lemma 5.6. For every formula $\alpha$, and any indices $\mu$ and $\nu$,

$$
\text { if } \mu, \nu:: \alpha \in S \text {, then } \mathcal{M}, \mu, \nu=\alpha \text {. if } \mu, \nu:: \neg \alpha \in S \text {, then } \mathcal{M}, \mu, \nu \mid \neq \alpha \text {. }
$$


Proof. Once again, a full proof involves a long structural induction. We only include the base cases, and the cases related to the F operator, as the others are essentially unchanged from Lemma 3.16.

If $\mu, \nu:: p \in S$, then, by definition of $\mathcal{M}, \nu \in V(p)$. So $\mathcal{M}, \mu, \nu \mid=p$.

If $\mu, \nu:: \neg p \in S$. Then, since $S$ is consistent, we know that $\mu, \nu:: p \notin S$. Furthermore, we know, from the consistency of $S$ as well as the fact that if a prefixed formula appears in $S$, so do the results of applicable tableau rules, that there is no $\sigma$ s.t. $\sigma, \nu:: p \in S$. Thus $\nu \notin V(p)$, and so $\mathcal{M}, \mu, \nu \not \models p$ and $\mathcal{M}, \mu, \nu \models \neg p$.

For the fixedly cases, we first consider the presence of $\mu, \nu:: \mathrm{F} \varphi \in S$. This implies the presence of $\sigma, \nu:: \varphi$ in $S$ for every $\sigma$ found in $S$. Thus, we have $\mathcal{M}, \sigma, \nu \models \varphi$ for all $\sigma$, and so $\mathcal{M}, \sigma, \nu \models \mathrm{F} \varphi$.

Finally, when $\mu, \nu:: \neg \mathrm{F} \varphi \in S$, then, by the fact that $S$ is prefix-complete, we have that there is some $\sigma$ s.t. $\sigma, \nu:: \neg \varphi \in S$, and so $\mathcal{M}, \sigma, \nu \models \neg \varphi$. However, this is sufficient to ensure that $\mathcal{M}, \mu, \nu \not \models \mathrm{F} \varphi$.

Corollary 5.7. If $\alpha$ is generally valid, it has a $\mathbf{K}+\mathbf{A}_{\mathbf{1}}+\mathbf{F}$ tableau proof.

Corollary 5.8. If $\alpha$ is diagonally valid, it has a $\mathbf{K}+\mathbf{A}_{\mathbf{2}}+\mathbf{F}$ tableau proof.

Proof. This proceeds in precisely the same manner as for $\mathbf{A}_{\mathbf{1}}$. The only difference is that when building our set $S$, we start with $0,0:: \neg \varphi$ rather than $0,1:: \neg \varphi$.

\section{$6 \quad$ Adding $\uparrow$}

Finally, in this section we will specify tableau rules for the $\uparrow$ operator, and demonstrate that these rules can straightforwardly be added to the rules of $\mathbf{K}+\mathbf{A}$ or $\mathbf{K}+\mathbf{A}+\mathbf{F}$.

The semantic condition for $\uparrow$ is:

$$
\mathcal{M}, w, v \models \uparrow \varphi \text { iff } \mathcal{M}, v, v \models \varphi
$$

Intuitively, $\uparrow$ allows us to store the current evaluation index as the reference index with the effect that subsequent @ operators will refer to this stored world, and not the original reference world. Thus, such an operator is required to formalize natural language sentences involving these types of shift of reference. ${ }^{11}$

\footnotetext{
${ }^{11}$ Cresswell [7] illustrates this need by way of the following example: "If it might have been that everyone actually rich was poor, then the economy would have been in bad shape". One can consult [7] for a more detailed discussion regarding the usefulness of this operator, as well as for more examples.
} 
The semantic condition naturally suggests the following tableau rules, for any $\mu$ and $\nu$ :

$$
\frac{\mu, \nu:: \uparrow \varphi}{\nu, \nu:: \varphi} \quad \frac{\mu, \nu:: \neg \uparrow \varphi}{\nu, \nu:: \neg \varphi}
$$

Since the $\uparrow$ operator introduces an apparatus for altering the first index, whenever we wish to add these rules, we must also, just like for $F$, also add the rule:

$$
\frac{\mu, \nu:: p}{\sigma, \nu:: p}
$$

When these three rules are added to the system $\mathbf{K}+\mathbf{A}$, we obtain the system we can call $\mathbf{K}+\mathbf{A}+\mathbf{X}$. If we add just the first two rules to $\mathbf{K}+\mathbf{A}+\mathbf{F}$, we get the system $\mathbf{K}+\mathbf{A}+\mathbf{F}+\mathbf{X}$.

As before, we have two different notions of proof, and these are straightforwardly altered so as to accommodate the new operator. Thus we will now talk about, for example, $\mathbf{K}+\mathbf{A}_{\mathbf{1}}+\mathbf{X}$ proofs and $\mathbf{K}+\mathbf{A}_{\mathbf{2}}+\mathbf{F}+\mathbf{X}$ proofs.

\subsection{Soundness}

Since we are again just adding new rules, proving soundness (and completeness in the next subsection), only involves amending the corresponding proofs for our previous systems. Specifically, we need a new version of Lemma 5.1, showing that the new rules preserve satisfiability.

Lemma 6.1. Given a satisfiable tableau, the tableau remains satisfiable after the application of one of the new rules for $\uparrow$.

Proof. Note that we just need to consider the rules for $\uparrow$ since the rule governing the indices of propositional variables is already included in Lemma 5.1 .

So let $\mathcal{T}$ be a tableau containing the satisfiable branch $\mathcal{B}$.

If $\mathcal{B}$ contains $\mu, \nu:: \uparrow \varphi$ we have that there is some $\mathcal{M}$ and $\theta$ such that $\mathcal{M}, \theta(\mu), \theta(\nu) \models \uparrow \varphi$. Thus $\mathcal{M}, \theta(\nu), \theta(\nu) \models \varphi$, and so $\mathcal{B} \cup\{\nu, \nu:: \varphi\}$ is satisfiable.

On the other hand, if $\mathcal{B}$ contains $\mu, \nu:: \neg \uparrow \varphi$ then $\mathcal{M}, \theta(\mu), \theta(\nu) \models \neg \uparrow \varphi$, and so $\mathcal{M}, \theta(\nu), \theta(\nu) \not \models \varphi$. Therefore, $\mathcal{B} \cup\{\nu, \nu:: \neg \varphi\}$ is satisfiable.

Corollary 6.2. The following soundness results follow: 
1. If $\varphi$ has an $\mathbf{K}+\mathbf{A}_{\mathbf{1}}+\mathbf{X}$ tableau proof, then it is generally valid.

2. If $\varphi$ has an $\mathbf{K}+\mathbf{A}_{\mathbf{2}}+\mathbf{X}$ tableau proof, then it is diagonally valid.

3. If $\varphi$ has an $\mathbf{K}+\mathbf{A}_{\mathbf{1}}+\mathbf{F}+\mathbf{X}$ tableau proof, then it is generally valid.

4. If $\varphi$ has an $\mathbf{K}+\mathbf{A}_{\mathbf{2}}+\mathbf{F}+\mathbf{X}$ tableau proof, then it is diagonally valid.

\subsection{Completeness}

For the proof of completeness we will just modify the proof of Lemma 5.6, the truth lemma used in the completeness of the logics with the fixedly operator. Note that if one wants a completeness proof for the system containing rules for $\uparrow$, but without fixedly, it is lemma 3.16 that needs to be altered. In this latter case, one needs to take some care as simply adding cases to the induction corresponding to the new rules will not be sufficient: one must also modify the truth lemma to encompass arbitrary indices in both positions, as in the proof of Lemma 5.6. Again, this will be rendered unproblematic by the presence of the rule prohibiting the presence, for example, of both $\mu, \nu:: p$ and $\rho, \nu:: \neg p$ in a maximally consistent and prefix-complete set.

Therefore, as before, we construct a maximally consistent (with respect to $\mathbf{K}+\mathbf{A}+\mathbf{F}+\mathbf{X}$ ) and prefix-complete set $S$, and let $\mathcal{M}$ be the model in which $W$ is the set of indices from $S, \mu R \nu$ iff $\nu=\mu$. $k$ for some $k$, and $v \in V(p)$ just in case $\mu, \nu:: p \in S$ for some $\mu$. Then we can prove the truth lemma, as before.

Lemma 6.3. For every formula $\alpha$, in the modal language containing @, F, and $\uparrow$, and any indices $\mu$ and $\nu$,

$$
\begin{aligned}
& \text { if } \mu, \nu:: \alpha \in S, \text { then } \mathcal{M}, \mu, \nu \models \alpha \\
& \text { if } \mu, \nu:: \neg \alpha \in S \text {, then } \mathcal{M}, \mu, \nu \not \alpha
\end{aligned}
$$

Proof. We only include the cases relevant for $\uparrow$.

Specifically, if $\mu, \nu:: \uparrow \varphi \in S$ then $\nu, \nu:: \varphi \in S$, and so $\mathcal{M}, \nu, \nu \models \varphi$, and so $\mathcal{M}, \mu, \nu \mid=\uparrow \varphi$.

In the second case, if $\mu, \nu:: \neg \uparrow \varphi \in S$ then $\nu, \nu:: \neg \varphi \in S$, and so $\mathcal{M}, \nu, \nu \not \models \varphi$, and $\mathcal{M}, \mu, \nu \not \models \uparrow \varphi$.

Corollary 6.4. This gives the following completeness results:

1. If $\alpha$ is generally valid, it has a $\mathbf{K}+\mathbf{A}_{\mathbf{1}}+\mathbf{X}$ tableau proof. 
2. If $\alpha$ is diagonally valid, it has a $\mathbf{K}+\mathbf{A}_{\mathbf{2}}+\mathbf{X}$ tableau proof.

3. If $\alpha$ is generally valid, it has a $\mathbf{K}+\mathbf{A}_{\mathbf{1}}+\mathbf{F}+\mathbf{X}$ tableau proof.

4. If $\alpha$ is diagonally valid, it has a $\mathbf{K}+\mathbf{A}_{\mathbf{2}}+\mathbf{F}+\mathbf{X}$ tableau proof.

\section{Conclusion}

Formal accounts of two-dimensional modal logics represent an interesting and growing area of philosophical logic. The results in this paper are intended to provide a foundation for inquiries in this area for those interested in tableau methods. Particularly, the results on the fixedly operator represent the most obvious addition to a previously underexplored area. We have presented doubly-indexed prefixed tableau systems for some basic modal logics supplemented with the actuality and fixedly operators, as well as the $\uparrow$ operator, and proved these systems sound and complete with respect to a two-dimensional semantics. In addition, it was shown that the standard decision procedures often used to establish decidability for tableau systems can be adapted in a straight-forward manner to the doubly-indexed case for the actuality operator.

There remains, however, a great deal of work to be done. Most obviously, the problem of various sorts of quantification presents itself, as does the issue of how to handle other two-dimensional operators. It should also be noted that many of the advantages of two-dimensionality can also be obtained via hybrid logics [2]. Thus, it may be natural to apply techniques from hybrid tableaux (e.g. [3]) to the two-dimensional case, and to investigate further their interconnectedness. However, we have refrained from such an investigation here as hybrid logics have expressive power far in excess of the two-dimensional systems considered in this paper, and the current project is to see how to produce tableaux using these more limited resources. We intend to explore these problems in future projects.

\section{Acknowledgements}

I would like to thank Giorgio Venturi, Paolo Maffezioli, Ed Mares, and Rob Goldblatt for their comments and feedback on various sections of this paper. I would also like to thank an anonymous referee, whose comments greatly improved the paper and its presentation, along with the editorial staff of this journal. 


\section{A Finite Model Property for F and $\uparrow$}

The usual techniques for constructing filtrations can be put to use here, with only minor modifications. We prove the results for the full logic (containing $\uparrow)$, but things would work fine were one only interested in various fragments.

Definition A.1. Let $\mathcal{M}=\langle W, R, V\rangle$ be a relational model, and $\Gamma$ a set of formulas in our language (with $\square, @, F, \uparrow$ ). Define the relation $\sim_{\Gamma}$ on $W$ by

$$
x \sim_{\Gamma} y \text { iff for all } \alpha \in \Gamma, \mathcal{M}, x, x=\alpha \text { iff } \mathcal{M}, y, y=\alpha
$$

Remark A.2. $\sim_{\Gamma}$ is an equivalence relation.

Let $[x]_{\Gamma}$, or just $[x]$, when the context is unambiguous, be the equivalence class of $x$.

Definition A.3 (Filtration). Let $\mathcal{M}=\langle W, R, V\rangle$ be a relational model and $\Gamma$ a set of formulas closed under subformulas. A filtration is a model $\mathcal{M}_{\Gamma}=\left\langle W_{\Gamma}, R_{\Gamma}, V_{\Gamma}\right\rangle$ such that:

$$
\begin{aligned}
& W_{\Gamma}:=\{[x] \mid x \in W\} ; \\
& \text { if } w R v \text { then }[w] R_{\Gamma}[v] ; \\
& \text { if }[w] R_{\Gamma}[v] \text { then, for all } \square \varphi \in \Gamma \text {, if } \mathcal{M}, w_{0}, w \models \square \varphi \text { then } \mathcal{M}, w_{0}, v \models \varphi ; \\
& V_{\Gamma}(p):=\{[x] \mid x \in V(p)\} \text { for all propositional letters in } \Gamma \text { (for those } \\
& \text { not in } \Gamma \text {, the function can be arbitrarily defined). (The definition of } \\
& \left.\sim_{\Gamma} \text { ensures that }[x] \in V_{\Gamma}(p) \text { iff }[y] \in V_{\Gamma}(p) \text { when } x \sim_{\Gamma} y .\right)
\end{aligned}
$$

We can often just assume we are working with a specific filtration, namely the quotient structure of $\mathcal{M}$ by $\sim_{\Gamma}$ (called the smallest filtration in [1]), with the accessibility relation defined by

$$
[x] R_{\Gamma}[y] \text { iff } \exists w \in[x], z \in[y] \text { s.t. } w R z
$$

Lemma A.4. When $\Gamma$ is a finite, subformula-closed set of formulas, then $\mathcal{M}_{\Gamma}$ is finite, with cardinality bounded by $|\wp(\Gamma)|$.

Proof. Define $g: W_{\Gamma} \rightarrow \wp(\Gamma)$ by

$$
g([x]):=\{\alpha \in \Gamma|M, x, x|=\alpha\}
$$

Assume that $x \sim_{\Gamma} y$. Then, for all $\alpha \in \Gamma, \mathcal{M}, x, x=\alpha$ iff $\mathcal{M}, y, y=\alpha$. Thus $g([x])=g([y])$, and so $g$ is well-defined. 
Furthermore, if $[x] \neq[y]$, then $\exists \alpha \in \Gamma$ s.t. (wlog) $\mathcal{M}, x, x \models \alpha$ but $\mathcal{M}, y, y \not \models \alpha$. Thus $\alpha \in g([x])$ but $\alpha \notin g([y])$. Hence, $g$ is injective.

Theorem A.5. Let $\mathcal{M}_{\Gamma}$ be a filtration with $\Gamma$ a subformula-closed set. Then, for all $\alpha \in \Gamma$ and all $x, y \in W$

$$
\mathcal{M}, x, y \models \alpha \text { iff } M_{\Gamma},[x],[y] \models \alpha
$$

Proof. In the case where $\alpha$ is some propositional variable $p, \mathcal{M}, x, y=p$ iff $y \in V(p)$ iff $[y] \in V_{\Gamma}(p)$ (by definition of $\mathcal{M}_{\Gamma}$ ) iff $\mathcal{M}_{\Gamma},[x],[y] \models p$.

The boolean cases are straightforward.

If $\alpha$ is of the form $\square \varphi$, then $\mathcal{M}, x, y \not \models \square \varphi$ implies the exists of some $w \in W$ s.t. $y R w$ and $\mathcal{M}, x, w \not \models \varphi$. Then, since $\varphi \in \Gamma, \mathcal{M}_{\Gamma},[x],[w] \not \models \varphi$. Also, $[y] R_{\Gamma}[w]$, by definition, and so $\mathcal{M}_{\Gamma},[x],[y] \not \models \square \varphi$.

In the other direction, $\mathcal{M}_{\Gamma},[x],[y] \not \forall \square \varphi$ implies that there is a $[w]$ such that $[y] R_{\Gamma}[w]$ and $\mathcal{M}_{\Gamma},[x],[w] \not \models \varphi$. This gives $\mathcal{M}, x, w \not \models \varphi$, and so $\mathcal{M}, x, y \forall \forall \square$ (from the definition of $\mathcal{M}_{\Gamma}$ ).

$\mathcal{M}, x, y \models @ \varphi$ iff $\mathcal{M}, x, x \models \varphi$ iff $(\mathrm{IH}) \mathcal{M}_{\Gamma},[x],[x] \models \varphi$ iff $\mathcal{M}_{\Gamma},[x],[y] \models$ @ $\varphi$.

$\mathcal{M}, x, y=\mathrm{F} \varphi$ iff $\mathcal{M}, w, y=\varphi$ for all $w \in W$ iff $(\mathrm{IH}) \mathcal{M}_{\Gamma},[w],[y] \models \varphi$ for all $[w] \in W_{\Gamma}$ iff $\mathcal{M}_{\Gamma},[x],[y] \models \mathrm{F} \varphi$.

$\mathcal{M}, x, y \models \uparrow \varphi$ iff $\mathcal{M}, y, y \models \varphi$ iff (IH) $\mathcal{M}[y],[y] \models \varphi$ iff (semantic definition of $\uparrow) \mathcal{M},[x],[y] \models \uparrow \varphi$.

Corollary A.6. Validity is decidable for $\mathbf{K}+\mathbf{A}_{\mathbf{1}}+\mathbf{F}+\uparrow$ and $\mathbf{K}+\mathbf{A}_{\mathbf{2}}+\mathbf{F}+\uparrow$.

Proof. For any formula $\alpha$, if $\neg \alpha$ is satisfiable it is satisfiable on a model of size $2^{n}$ where $n$ is the number of subformulas of $\alpha$. There are only finitely many such models (up to isomorphism).

\section{References}

[1] P. Blackburn, M. De Rijke, and Y. Venema. Modal Logic. Cambridge University Press, 2001.

[2] P. Blackburn and M. Marx. Remarks on Gregory's "actually" operator. Journal of Philosophical Logic, 31:281-288, 2001.

[3] T. Bolander and P. Blackburn. Termination for hybrid tableaus. Journal of Logic and Computation, 17(3):517-554, 2007. 
[4] P. Bricker. Quantified modal logic and the plural de re. Midwest Studies in Philosophy, 14:372-394, 1989.

[5] D. Chalmers. Two-dimensional semantics. In E. Leopre and B. Smith, editors, Oxford Handbook of Philosophy of Language, pages 574-606. Oxford University Press, New York, 2006.

[6] F. Correia. Modality, quantification, and many vlach-operators. Journal of Philosophical Logic, 36:473-488, 2007.

[7] M.J. Cresswell. Entities and Indicies. Kluwer, Dordrecht, The Netherlands, 1990.

[8] M. Davies. Meaning, Quantification, Necessity. Routledge, London, 1981.

[9] M. Davies. Reference, contingency, and the two-dimensional framework. Philosophical Studies, 118:83-131, 2004.

[10] M. Davies and L. Humberstone. Two notions of necessity. Philosophical Studies, 38:1-30, 1980.

[11] G. Evans. Reference and contingency. The Monist, 62:161-189, 1979.

[12] M. Fitting. Tableau methods of proof for modal logics. Notre Dame Journal of Formal Logic, 13:237-247, 1972.

[13] M. Fitting. Proof Methods for Modal and Intuitionistic Logics. D. Reidel Publishing Company, Dordrecht, Holland, 1983.

[14] M. Fitting. Proof theory for modal logic. In P. Blackburn, J. Van Benthem, and F. Wolter, editors, Handbook of Modal Logic. Elsevier, Amsterdam, The Netherlands, 2007.

[15] M. Fitting and R. Mendelsohn. First-Order Modal Logic. Kluwer, Dordrecht, The Netherlands, 1998.

[16] G. Forbes. Languages of Possibility. Blackwell, Oxford, 1989.

[17] P. Fritz. A logic for epistemic two-dimensional semantics. Synthese, 190:1753-1770, 2013.

[18] R. Gore. Tableau methods for modal and temporal logics. In M. D'Agostino, D. Gabbay, R. Hähnle, and J. Posegga, editors, Handbook of Tableau Methods. Kluwer, Dordrecht, The Netherlands, 1999.

Australasian Journal of Logic (13:7) 2016, Article no. 1 
[19] D. Gorín and L. Schröder. Narcissists are easy, stepmothers are hard. In FoSSaCS 2012, volume 7213 of LNCS, pages 240-254. Springer, 2012.

[20] D. Gregory. Completeness and decidability results form some propositional modal logics containing "actually" operators. Journal of Philosophical Logic, 2:77-96, 1973.

[21] A. Hazen, B. Rin, and K. Wehmeier. Actuality in propositional modal logic. Studia Logica, 101:487-503, 2013.

[22] H. Hodes. Some theorems on the expressive limitations of modal languages. Journal of Philosophical Logic, 13:13-26, 1984.

[23] H. Kamp. Formal properties of 'now'. Theoria, 37:227-273, 1972.

[24] G. Restall. A cut-free sequent system for two-dimensional modal logic, and why it matters. Annals of Pure and Applied Logic, 163:1611-1623, 2012 .

[25] K. Segerberg. Two-dimensional modal logic. Journal of Philosophical Logic, 30:57-78, 2001.

[26] T. Sider. Logic for Philosophy. Oxford University Press, 2010.

[27] S. Soames. Reference and Description: The Case against TwoDimensionalism. Princeton University Press, Princeton, 2004.

[28] R. Stalnaker. Assertion revisited: On the interpretation of twodimensional modal semantics. Philosophical Studies, 118:299-322, 2004.

[29] B. ten Cate and M. Franceschet. On the complexity of hybrid logics with binders. In Computer Science Logic, CSL 2005, volume 3634 of LNCS, pages 339-354. Springer, 2005.

[30] F. Vlach. 'Now' and 'Then': A Formal Study in the Logic of Tense Anaphora. PhD thesis, University of California, Los Angeles, 1973. 